\title{
Causes de mortalité des petits ruminants sur le plateau du Sankaran-G uinée en 1992-1993
}

Mots-clés

Ovin Djallonké - Caprin Kirdi Mortalité - Saison - Inanition - Poids à la naissance - Conduite du troupeau Parasite - Guinée.

\author{
M. Mourad ${ }^{1}$ I.B. Baldé 1
}

\begin{abstract}
Résumé
Cette étude a eu pour objet d'identifier les principales causes de mortalité de 83 ovins et 124 caprins enregistrées sur le plateau du Sankaran, FaranahGuinée, en 1992-1993. Les mortalités les plus fortes, 43,8 et 39,1 p. 100, ont été observées respectivement chez les agneaux et les chevreaux de 3 à 120 jours. Les pertes les plus nombreuses pendant la saison humide et la saison sèche froide étaient associées aux effets inanition-poids léger à la naissancemauvaise gestion. Les parasites internes et externes constituaient une cause importante de la mortalité chez les jeunes après sevrage et cela durant toute l'année. L'absence de soins suffisants était également à l'origine de cas de mortalité.
\end{abstract}

\section{INTRO DUCTION}

Situé en Haute Guinée, le centre de Faranah a été créé pour mener une recherche adaptative ou appliquée en vue d'identifier les contraintes qui pèsent sur l'élevage et mettre à la disposition des éleveurs des techniques simples et peu coûteuses.

La mortalité est l'un des principaux facteurs qui freinent la productivité numérique et la rentabilité économique des élevages ovins et caprins dans la zone tropicale. Des travaux réalisés par plusieurs chercheurs ont montré des pertes causées par la mortalité des petits ruminants entre 13 et 68,4 p. 100 (Chawla et Bhatnager en Inde (1), Ndamukong au Cameroun (6), Njau et coll. en Ethiopie (7), Mourad en Egypte (5) et Lancelot et coll. au Tchad (4)).

Dans cette étude, les principales causes de mortalité des ovins Djallonké et des caprins de race Naine Africaine ont été identifiées par des examens cliniques, l'autopsie des cadavres et des analyses de laboratoire.

\section{MATERIEL ET METHODES}

De 1992 à 1993, 15 troupeaux mixtes choisis dans les élevages présentant un effectif total de 346 ovins (avec une moyenne de $23,07 \pm 1,43$ ) et 352 caprins (avec une moyenne de 23,47 $\pm 1,76$ )

1. Centre de Recherche Zootechnique de Faranah (CRZF/IRAG), BP 1523, Conakry, Guinée ont été individuellement identifiés. La localisation des troupeaux est indiquée à la figure 1. Des passages hebdomadaires ont été effectués. Trois groupes d'âge ont été constitués : de la naissance à 3 jours, de 3 à 120 jours (sevrage) et plus de 120 jours. Le nombre de morts par espèce et par catégorie a été enregistré. L'autopsie a été pratiquée dans les $24 \mathrm{~h}$ qui ont suivi la mort et a permis l'examen de certains organes : langue, poumons, coeur, foie, estomac, gros intestin et intestin grêle. Cinq périodes ont été considerées au cours de l'année : saison sèche froide (décembre, janvier et février) ; saison sèche chaude (mars et avril) ; début de la saison humide (mai et juin) ; saison humide (juillet, août et septembre) ; début de la saison froide (octobre et novembre). Le taux de mortalité brut annuel (Tm) avant sevrage est représenté formellement par un pourcentage calculé comme suit :

$$
\mathrm{Tm}=\mathrm{M} / \mathrm{E} \times 100
$$

où $\mathrm{M}$ est le nombre de nouveaux-nés morts entre la naissance et le sevrage et E l'effectif annuel total de nouveaux-nés.

Le taux de mortalité brut annuel après sevrage a été calculé comme suit :

$$
\mathrm{Tm}=\mathrm{M} / \mathrm{E} \times 100
$$

où $\mathrm{M}$ est le nombre annuel des morts après sevrage et É l'effectif moyen du troupeau $(E ́ \mathrm{E}=\mathrm{E} 1+\mathrm{E} 2+\mathrm{E} 3 / 3)$ avec E1, E2 et E3 représentant les effectifs du troupeau au début, au milieu et à la fin de l'année.

Le taux de mortalité brut saisonnier avant sevrage a été calculé en utilisant l'équation (1) tandis que celui après sevrage a été obtenu 


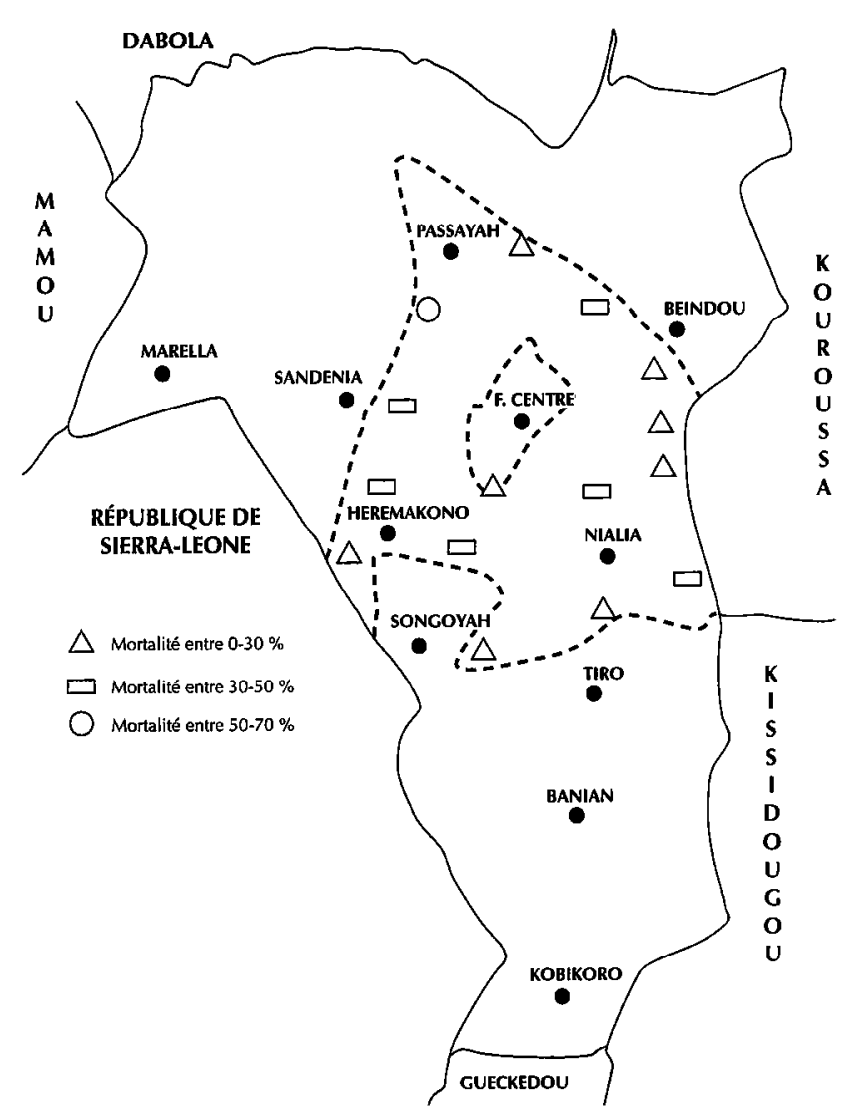

Figure 1 : localisation et taux de mortalité des 15 troupeaux mixtes (ovins-caprins) suivis sur le plateau du Sankaran, Faranah, 1992-1993.

en appliquant l'équation (2) au cours d'une saison. Toutes fautes imputables aux éleveurs (surdosages de produits vétérinaires, morsures des chiens, accidents, défaut de surveillance des jeunes...) causant la perte d'un animal ont été groupées sous le terme « mauvaise gestion ». Le test de student (test-t) qui suit le test d'homogénéité de deux variances a été effectuć pour tcstcr la signification de la différence entre les deux moyennes de deux espèces (ovins et caprins) pour ce qui concerne les mortalités dues à l'inanition (production laitière insuffisante).

\section{RESULTATS}

Entre février 1992 et janvier 1993, 49 cas de mortalité ont été enrcgistrćs parmi les agneaux avant sevrage (tableau I). Ceci représente un taux de mortalité brut annuel de 38,6 p. 100 . Chez les chevreaux de la même catégorie, ce taux est de 65,5 p. 100 à la même période. La mortalité brute saisonnière chez les agneaux (figure 2) est plus élevée pendant la saison humide et la saison sèche froide (respectivement 25,6 et 22,5 p. 100). Ce taux est de 29,4 et 27,5 p. 100 chez les chevreaux avant sevrage. Chez les ovins, 34 morts ont été enregistrées après sevrage, ce qui représente un taux brut annuel de 10,5 p. 100 , alors que chez les caprins de la même catégorie il est de 20,0 p. 100 (tableau II). Le taux de mortalité brut saisonnier après sevrage (figure 3 ) est plus élevé en saison humide et en saison sèche froide aussi bien chez les caprins (13,3 et 6,1 p. 100) que chez les ovins $(5,2$ et 3,8 p. 100).

Les principales causes de mortalité chez les ovins et les caprins avant sevrage sont reportées au tableau I, celles après sevrage au tableau II. L'inanition, la mauvaise gestion et le poids léger à la naissance ont causé à eux trois plus de 50 p. 100 de mortalité chez les jeunes : respectivement $28,6,24,5$ et 4,08 p. 100 des cas de mortalité chez les agneaux et $39,8,12,1$ et 3,68 p. 100 des cas de mortalité chez les chevreaux.

Le taux de mortalité dû à l'inanition est significativement $(\mathrm{P}<0,05)$ plus élevé chez les chevreaux que chez les agneaux. Avant sevrage, les parasites internes ont causé 16,3 et 7,2 p. 100 des cas de mortalité, respectivement chez les agneaux et les chevreaux. D'aulres causes moins importantes ont été identifiées chez les deux espèces comme indiqué au tableau I.

Après sevrage, les parasites internes, externes et la mauvaise gestion étaient responsables de la plupart des cas de mortalité enregistrés chez les ovins $(67,66$ p. 100) et chez les caprins $(60,01$ p. 100$)$. Des causes secondaires de mortalité sont notées dans le tableau II.

\section{DISCUSSION}

Les comparaisons avec les données de la littérature doivent être prises en considération avec prudence, des différences, qui ne sont pas toujours clairement explicitées dans le mode de calcul, pouvant varier d'un auteur à l'autre.

Dalton (2) a trouvé un taux de mortalité de 22 p. 100 chez les ovins d'Australie. Njau et coll. (7) ont noté un taux de 33,3 p. 100 chez les ovins des hauts plateaux éthiopiens. Ces taux indiqués par deux auteurs sont supérieurs à ceux trouvés chez les agneaux Djallonké, et inférieurs chez les chevreaux de la race Naine Africaine.

Au Cameroun, Ndamukong (6) a observé chez les ovins locaux un taux de mortalité de 68,4 p. 100 , supérieur à celui obtenu dans cette étude chez les moutons Djallonké (38,6 p. 100) et proche de celui obtenu chez les chèvres Naines Africaines $(65,5$ p. 100 entre la naissance et le sevrage). Chawla et Bhatagar (1) ont obtenu des résultats similaires en Inde, 44,9 p. 100 entre la naissance et un mois d'âge. Les taux de mortalité relevés dans cette étude sont supérieurs à ceux indiqués par Mourad (5) en Egypte chez les chevreaux Zaraibi et Alpins français (13 et 16 p. 100).

Plus de 50 p. 100 des cas de mortalité enregistrés chez les ovins Djallonké et chez les caprins Nains Africains pour les troupcaux étudiés sont dus principalement aux effets inanition-poids léger à la naissance-mauvaise gestion. D'après Njau et coll. (7), cet effet ne représente que 7 p. 100 des cas de mortalité chez les agneaux locaux d'Ethiopie. En outre, Lancelot et coll. (4) estiment que la peste des petits ruminants, le poids léger à la naissance, l'insuffisance d'espace dans la bergerie sont les principaux facteurs de risque de la mortalité chez les jeunes. Les résultats de Haughey (3) sont proches de ceux de cette étude : 65 p. 100 de pertes ovines périnatales en $\Lambda$ ustralie liées aux effets inanition-mauvais soins maternels-exposition aux maladies. Selon Njau et coll. (7), ce problème s'explique par le manque de contrôle de la saillie qui entraine l'accouplement précoce des femelles et la non complémentation de la ration des femelles gestantes et allaitantes.

La diarrhée est responsable de 10,2 et 8,4 p. 100 des cas de mortalité, respectivement chez les agneaux et les chevreaux, mais l'étiologie n'a pas été étudiée. Njau et coll. (7) ont noté que seulement 1 p. 100 des cas de mortalité chez les agneaux éthiopiens de race locale est dû à la diarrhée. 


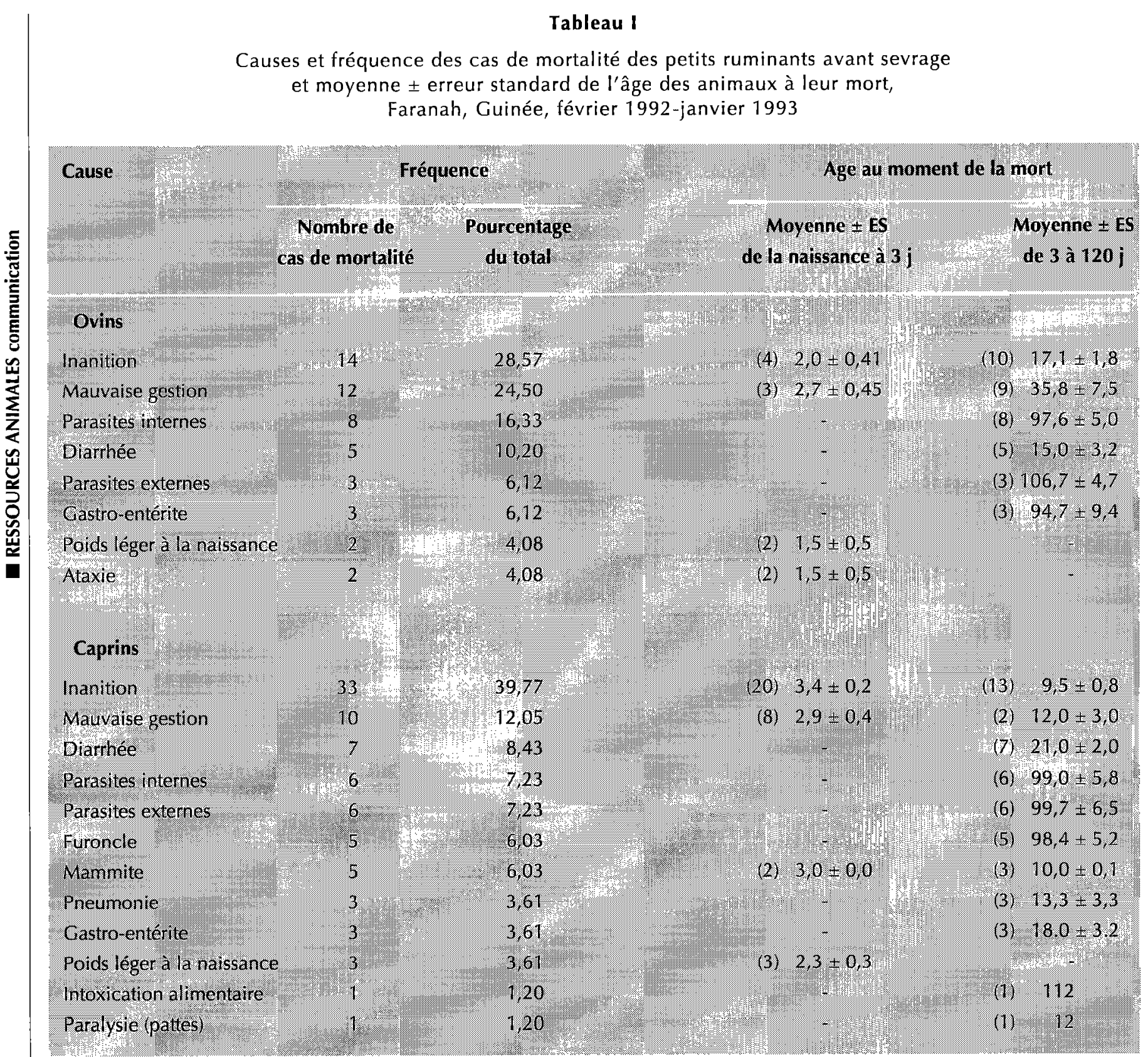

Les chiffres entre parenthèses représentent les effcctifs par catćgoric

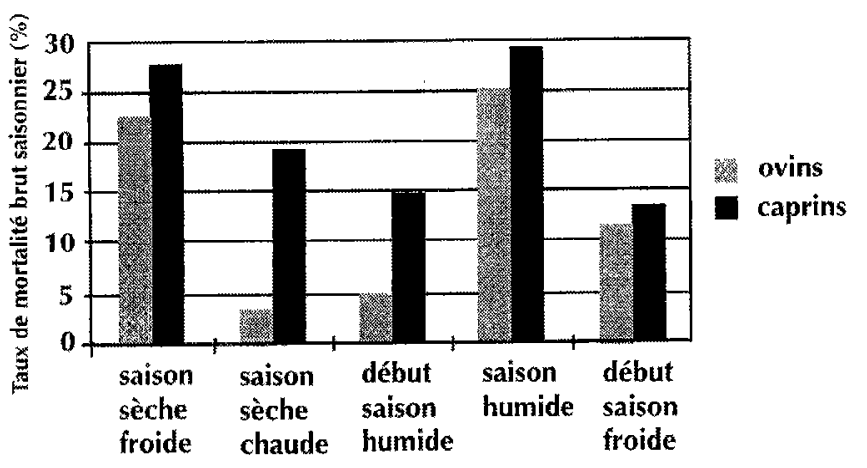

Figure 2 : taux de mortalité bruts saisonniers chez les ovins et les caprins de la naissance au sevrage à Faranah, Guinée, février 1992-janvier 1993.

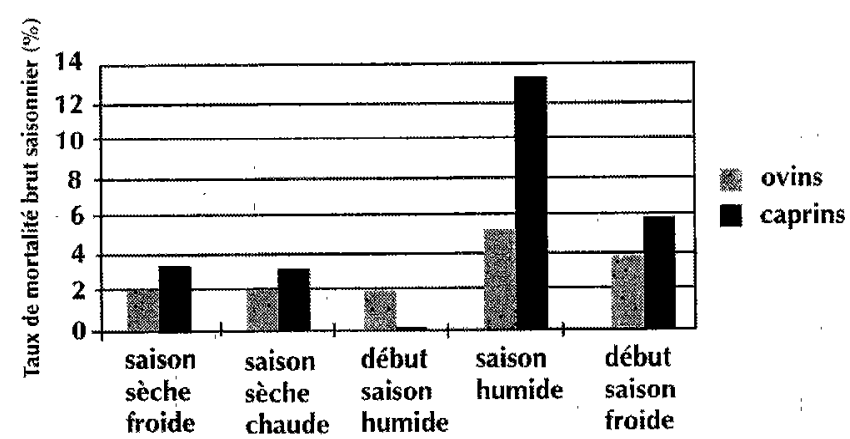

Figure 3 : taux de mortalité bruts saisonniers chez les ovins et les caprins après sevrage à Faranah, Guinée, février 1992-janvier 1993. 
Tableau II

Cause et fréquence des cas de mortalité des petits ruminants après sevrage et moyenne \pm erreur standard de l'âge des animaux à leur mort, Faranah, Guinée, février 1992-janvier 1993

\begin{tabular}{|c|c|c|c|}
\hline \multirow[t]{2}{*}{ Cause } & \multicolumn{2}{|c|}{ Fréquence } & \multirow{2}{*}{$\begin{array}{c}\begin{array}{c}\text { Age } \\
\text { au moment } \\
\text { de la mort } \\
\text { (mois) }\end{array} \\
\begin{array}{c}\text { Moyenne } \\
\pm \mathrm{ES}\end{array}\end{array}$} \\
\hline & $\begin{array}{c}\text { Nombre } \\
\text { de cas } \\
\text { de mortalité }\end{array}$ & $\begin{array}{l}\text { Pourcentage } \\
\text { du total }\end{array}$ & \\
\hline \multicolumn{4}{|l|}{ Ovins } \\
\hline Parasites internes & 10 & 29,41 & $21,0 \pm 5,2$ \\
\hline Parasites externes & 7 & 20,60 & $30,0 \pm 8,2$ \\
\hline Mauvaise gestion & 6 & 17,65 & $14,0 \pm 5,7$ \\
\hline Pneumonie & 4 & 11,65 & $11,4 \pm 5,2$ \\
\hline Boiterie & 2 & 5,88 & $9,5 \pm 0,1$ \\
\hline Gastro-entérite & 1 & 2,94 & 38,0 \\
\hline Cœnurose & 1 & 2,94 & 11,0 \\
\hline $\begin{array}{l}\text { Peste des } \\
\text { petits ruminants }\end{array}$ & 1 & 2,94 & 24,0 \\
\hline Charbon & 1 & 2,94 & 14,0 \\
\hline Pasteurellose & 1 & 2,94 & 25,0 \\
\hline \multicolumn{4}{|l|}{ Caprins } \\
\hline Parasites internes & 13 & 28,89 & $22,1 \pm 3,7$ \\
\hline Mauvaise gestion & 8 & 17,78 & $19,9 \pm 5,3$ \\
\hline Parasites externes & 6 & 13,34 & $19,0 \pm 5,1$ \\
\hline $\begin{array}{l}\text { Peste des } \\
\text { petits ruminants }\end{array}$ & 4 & 8,89 & $36,8 \pm 8,8$ \\
\hline Gastro-entérite & 3 & 6,67 & $70,0 \pm 1,5$ \\
\hline Paralysie (pattes) & 2 & 4,44 & $9,0 \pm 2,0$ \\
\hline Balano-prostite & 2 & 4,44 & $14,0 \pm 1,0$ \\
\hline Furoncle & 1 & 2,22 & 24,0 \\
\hline Pasteurellose & 1 & 2,22 & 29,0 \\
\hline Charbon & 1 & 2,22 & 18,0 \\
\hline
\end{tabular}

\section{CONCLUSION}

Cette étude met en évidence les effets inanition-poids léger à la naissance-mauvaise gestion qui affectent lourdement la survie des petits ruminants dans la zone d'investigation. Cet effet, responsable de plus de 50 p. 100 des cas de mortalité entre la naissance et le sevrage, peut être éliminé par des actions zootechniques. Les plus grandes pertes s'observent entre 3 et 120 jours $(45,8$ et 39,1 p. 100) chez les deux espèces, et sont plus importantes en saison humide et en saison sèche froide.

Des solutions sont proposées pour améliorer génétiquement la production laitière des femelles et orienter les éleveurs vers de meilleurs modes de conduite en vue de planifier la saison de mises bas. Il est par conséquent recommandé d'éviter les mises bas en saison humide et en saison sèche froide. Les soins vétérinaires aux nouveaux-nés semblent importants pour permettre la baisse du taux de mortalité et assurer la rentabilité économique des élevages de petits ruminants.

\section{Remerciements}

Les auteurs remercient Dr Gnagna Gbanamou, directeur du Centre pour son apport inestimable dans la réalisation de ce travail.

\section{BIBLIO GRAPHIE}

1. CHAWLA D.S., BHATNAGER D.S., 1988. Factors affecting mortality in goats. Indian J. Hered., 19: 18-23.

2. DALTON D.C., 1979. Sources of perinatal mortality. In: Proc. 29th Lincoln College Farmer's Conference, Canterbury, $\mathrm{New}$ Zealand, May 19-21, 1979

3. HAU GHEY K.C., 1983. New insights into rearing failure and perinatal lamb mortality. In: Sheep production and preventive medicine. Sidney, Australia, U niversity of Sidney, p. 135-147. (Proceedings N 0. 67)

4. LAN CELOT R., LESCO URRET F., FAYE B., 1995. Multilevel modelling of pre-weaning kid mortality during the cold, dry season 1991-1992 in the outskirts of N 'D jaména, Chad. Prev. vet. Med., 24: 171-186.

5. MOURAD M., 1993. Reproductive performance of Alpine and Zaraibi goats and growth of their first cross in Egypt. Small Ruminant Res., 12: 379-384.

6. NDAMUKONG K.J.M., 1989. L'élevage traditionnel de moutons et de chèvres en province Nord-ouest du Cameroun. In: Proc. African small ruminant research and development, Bamenda, Cameroon, January 18-25, 1989. Addis Ababa, Ethiopia, ILCA, 578 p.

7. NJAU B.C., KASALI O.B., SCHOLTENS R.G., MESFIN DEGEFA. 1988. Etudes sur le terrain et en laboratoire des causes de la mortalité ovine dans les hauts plateaux éthiopiens. Addis Abeba, Ethiopie, CIPEA, p. 25-29. (Bulletin $n^{\circ} 31$ )

Reçu le 2.9.96, accepté le 2.6.97 


\section{Summary}

Mourad M., Baldé I.B. Causes of small ruminant mortality on the Sankaran-G uinea plateau in 1992-93

The aim of this investigation was to identify the main causes of mortality of 83 sheep and 124 goats which were recorded in the plateau of Sankaran, Faranah, in 1992-93. The highest mortality rates, 43.8 and 39.1, were observed in lambs and kids, respectively, between 3 and 120 days of age. The heaviest losses during the wet and the dry cold seasons were linked to three causes, starvation-light birth weight-bad livestock management. Internal and external parasites were an important cause of mortality in the young after weaning, all year round. Insufficient animal care was also at the root of mortality cases.

Key words: Djallonke sheep - Kirdi goat - Mortality - Season Starvation - Birth weight - Livestock management - Parasite Guinea.

\section{Resumen}

Mourad M., Baldé I.B. Causas de mortalidad en los pequeños rumiantes en la meseta de Sakarán-Guinea, en 1992-1993

El presente estudio se propone identificar las principales causas de mortalidad de 83 ovinos y 124 caprinos registradas en la meseta de Sankarán, Faranah-Guinea, en 1992-1993. Las mortalidades más fuertes $(43,8$ y $39,1 \%)$ se observaron respectivamente en los corderos y los cabritos de 3 a 120 días. Las pérdidas más importantes durante la estación húmeda y la estación seca fría se asociaron al complejo inanición-bajo peso al nacimiento-mala gestación. Los parásitos internos y externos constituyen una causa importante de mortalidad en los jóvenes después del destete y esto durante todo el año. La ausencia de cuidados suficientes se encuentra igualmente al origen de los decesos.

Palabras clave: O vino Djallonke - Caprino Kirdi - Mortalidad Estación - Inanición - Peso al nacimiento - Manejo del ganado - Pasasito - Guinea. 